\title{
Importance of Accurate Diagnosis in Pyoderma Gangrenosum
}

\author{
Yoon Soo Kim, \\ Han Kyeol Kim, \\ Yea Sik Han \\ Department of Plastic and Reconstructive \\ Surgery, Kosin University College of Medicine, \\ Busan, Korea
}

No potential conflict of interest relevant to this article was reported.

\begin{abstract}
Pyoderma gangrenosum is a rare inflammatory reactive dermatosis marked by painful cutaneous ulcers. Diagnosis of pyoderma gangrenosum is usually made based on past medical history and after the exclusion of other possible diseases based on the clinical manifestations of the lesion. Diseases that show rapid progression to necrosis and that should not be misdiagnosed as pyoderma gangrenosum include malignant neoplasms and necrotizing fasciitis. Immunosuppressive agents such as steroids and cyclosporine are considered first-line therapy. Surgical removal of the necrotic tissues is contraindicated, as it may further induce immune reaction and promote ulcer to enlarge. Here, we present a case to encourage plastic surgeons to consider pyodermagangrenosum in the differential diagnosis of idiopathic ulcers. Satisfactory outcomes for patients with pyodermagangrenosum may be expected when using steroids and immunosuppressive agents during the early stage of the disease.
\end{abstract}

Keywords: Pyoderma gangrenosum / Necrotizing fasciitis / Malignant neoplasms

\section{INTRODUCTION}

Pyoderma gangrenosum $(\mathrm{PG})$ is a rare inflammatory reactive dermatosis marked by painful cutaneous ulcers [1] and is classified into four types: 1) ulcerative; 2) pustular; 3) bullous; and 4) vegetative. The ulcerative type is the most common, with the remaining three types being rare. The etiology of PG is not clearly understood, but the inflammatory disorder is relatively common in patients with inflammatory bowel diseases and in patients with immunological diseases such as rheumatoid arthritis [2]. There are typical and atypical forms of PG. Typical PG usually develops as a lesion with reddish violet margins on anterior surface of lower extremities, and has a pattern of progressive ulceration accompanied by pain. In contrast, atypical PG includes hematic abscesses

\section{Correspondence: Yea Sik Han}

Department of Plastic Surgery, Kosin University College of Medicine, 262 Gamcheon-ro, Seo-gu, Busan 602-702, Korea

E-mail: hanplastic1@naver.com

Received November 14, 2013 / Revised July 31, 2014 / Accepted Ocbober 25, 2014 and usually develops in the head, cervical area, and upper extremities [3]. Due to the wide variety of clinical manifestations of PG, diagnosis is usually made after other diagnoses have been excluded. This report describes the diagnosis and treatment of a patient with PG who presented with an extensive progressive ulcer in the cervical area.

\section{CASE REPORT}

A 33-year-old male patient had developed a $0.5 \times 0.5 \mathrm{~cm}$ pyogenic papule in the left cervical area one month prior to presentation. At that time, the patient had been evaluated at a community hospital where the lesion was diagnosed as cellulitis and was given topical steroid and IV antibiotic treatment for two weeks without appropriate response. In the subsequent two weeks leading up to presentation at our hospital, the lesion continued to grow in size while the patient began to experience consistent fever and chills.

Initial physical examination revealed a painful $9 \times 4 \mathrm{~cm}$ ulcerative lesion in the left cervical area (Fig. 1). The patient reported a 
history of chemical burn on the left chin 13 years earlier and three scar revision procedures had been performed for keloid. The patient had been receiving local steroid injection therapy up until three years before the development of ulcer.

Upon admission, debridement and bacterial culture tests were performed to exclude the potential for necrotizing fasciitis. Histopathologic examinations were conducted to assess the possibility of malignant neoplasms. The white blood cell (WBC) count increased to $17.56 \times 10^{3}$, and the patient continued to experience febrile episodes $\left(39^{\circ} \mathrm{C}-40^{\circ} \mathrm{C}\right)$ two to three times a day. Findings characteristic of a widely spreading ulcer were observed. No microorganisms were identified in the wound culture. The histology of wound sample demonstrated papillary proliferation on kerati-

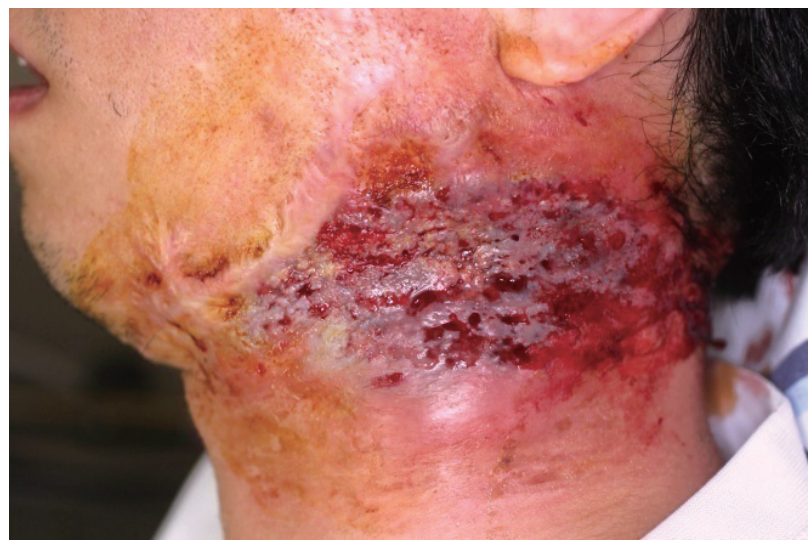

Fig. 1. A 33-year-old man with multiple ulcerative lesions around a reddish-purple area on the left lateral neck $(9 \times 4 \mathrm{~cm}$ in diameter).

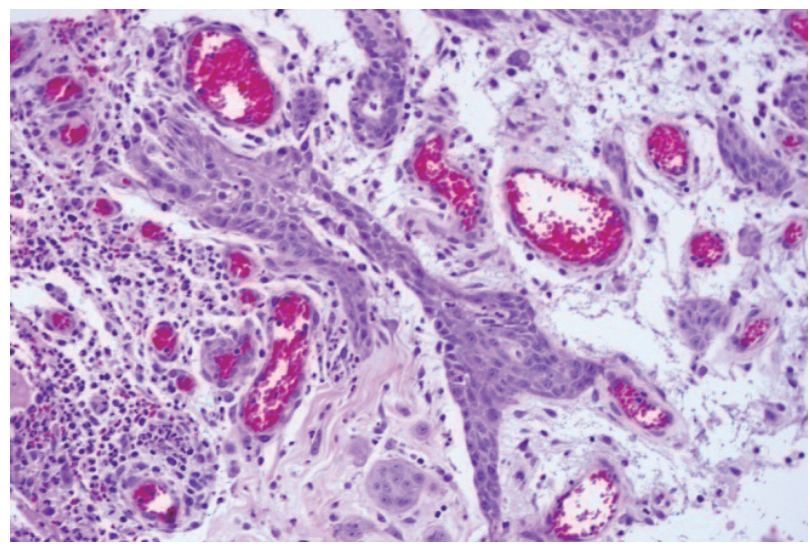

Fig. 2. Histopathologic image of the specimen from the ulcerative lesion $(\mathrm{H} \& \mathrm{E}, \times 200)$. The image shows neither stromal invasion nor cellular anaplasia, but rather papillary proliferation of keratinized squamous epithelium with significant chronic inflammation. nized squamous epithelium with significant chronic inflammation. The possibility of malignant neoplasms could not be excluded, although no stromal invasion or cellular an aplasia was observed (Fig. 2).

In cooperation with the pathology department, additional debridement was performed 8 days after admission. No necrotic findings were observed in the platysma fascia intraoperatively, and no cancer cells could be identified in the marginal ulcer area in frozen sections. Despite the consistent fever and increases in WBC count, bacteria were not observed in the ulcer or blood culture. Acute inflammation was confirmed based on the clinical manifestations of a progressive skin ulcer with a reddish violet margin and on tissue samples. Accordingly, PG was suspected over the possibility of malignant neoplasms (Fig. 3). Intravenous dexamethasone $(10 \mathrm{mg} /$ day) was administered, and the ulcer margin did not extend thereafter. The febrile episodes discontinued, and blood WBC count stabilized $\left(6.83 \times 10^{3}\right)$. After five days, IV dexamethasone was replaced with oral prednisolone (10 mg/day) for two weeks. The lesion stabilized approximately 15 days after starting the prednisolone regimen (Fig. 4). The defective area was reconstructed using a meshed split-thickness skin graft. A satisfactory outcome was confirmed two months after surgery (Fig. 5).

PG is a rare, progressive, ulcerative skin disease that was first reported in 1908 by Louis Broc. It is not clearly understood, but may be associated with a defective immune system. About 40\%-

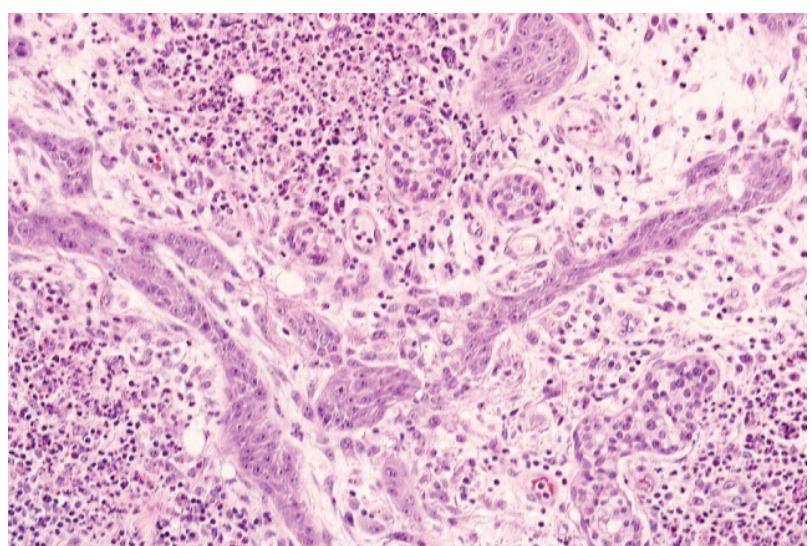

Fig. 3. Histopathologic image of the specimen from a lesion on the left lateral neck (H\&E, $\times 200)$, showing neutrophilic perivascular infiltration throughout the whole field, with the absence of squamous cell carcinoma or other malignancy. 


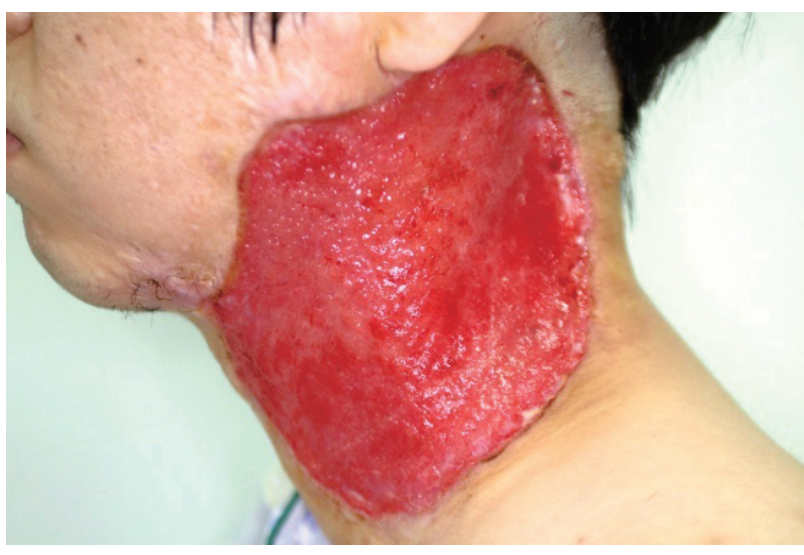

Fig. 4. Preoperative view. Two weeks following the oral administration of prednisolone (Solondo $10 \mathrm{mg} /$ day).

$50 \%$ of cases are accompanied by rheumatoid arthritis or inflammatory bowel diseases such as ulcerative colitis or Crohn's disease [2]. The inflammatory skin disorder usually develops in the anterior surface of lower extremities, but can develop in any area of the body including the head, cervical, abdominal and inguinal regions [4]. Typical PG usually develops rapidly with associated pain, whereas atypical PG usually develops in the head, cervical region, and superficial upper extremities with hematic abscesses [5]. The diagnosis of PG is made usually based on past medical history and clinical manifestations of the lesion, after other possible diagnoses have been excluded. Many patients report a past history of skin injury. The lesion appears as small papules, erythema nodosum, or a red plate, and progresses to hematic abscesses and necrotic lesions. Histological tests show nonspecific findings of extensive neutrophil invasion and proliferation in the surrounding blood vessels and tissues. Epithelial necrosis is also observed in PG [6]. The differential diagnosis for PG should include malignant neoplasms and necrotizing fasciitis.

Necrotizing fasciitis develops due to fulminant infection of fat cells, blood vessels, and nerves of the superficial fascia and subcutaneous tissues, leading to eventual skin necrosis. Since the fatality rate of necrotizing fasciitis is high, early surgical treatments and use of antibiotic agents are required [7].

Immunosuppressive agents such as steroids and cyclosporine are considered as first-line therapy for PG. Surgical removal of the necrotic tissues is contraindicated, as it may further induce im-

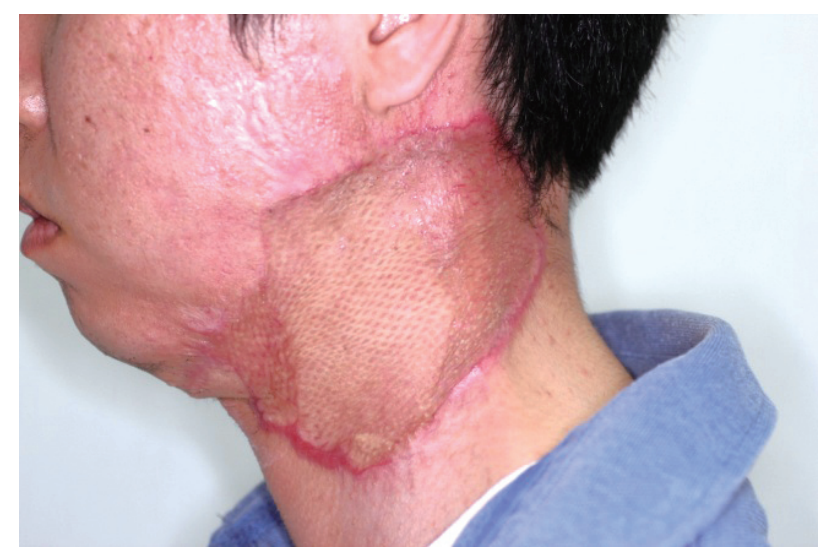

Fig. 5. Postoperative view 2 months after surgery. Appearance of the pyoderma gangrenosum lesion on the left lateral neck two months following skin grafting. The grafted skin was successfully tolerated, and the wound was stabilized.

mune reaction and promote ulcer to enlarge. Davis et al. [8] reported a successful case of necrotic tissue debridement and skin grafting followed by hyperbaric oxygen therapy. The authors hypothesized that hyperbaric oxygen therapy prevented the lack of arterial blood supply and consequent delay in wound healing, which is caused by the perivascular inflammation. However, surgical treatment for PG remains controversial. High fever and progressive ulceration with severe pain may indicate the need for earlier surgical debridement. However, the lesion continuously expanded in our patient. Only one week after admission, the patient was diagnosed with PG when the work up returned negatively for both necrotizing fasciitis and malignancy.

PG is a nonspecific disease with low prevalence as well as a diagnosis of exclusion in the differential company of necrotizing fasciitis. Furthermore, plastic surgical reconstruction of the lesion is challenging due to its rapid progression. Therefore, early diagnosis is important to prevent further acceleration of wound margin. As it was with the patient reported here, clinical suspicion for PG maybe low in patients with unexplainable ulcer without any history suggestive of an underlying systematic inflammatory disorder such as Crohn's disease or rheumatoid arthritis. Plastic surgeons should include PG in the differential diagnosis of idiopathic ulcers, and satisfactory outcomes may be expected using steroids and immunosuppressive agents in the early stages of disease. 


\section{REFERENCES}

1. Nam DH, Kim JH, Lee YM. A case of generalized pyoderma gangrenosum involving scalp and face. J Korean Cleft Palate-Craniofac Assoc 2011;12:53-7.

2. Bundino S, Zina AM. Pyoderma gangrenosum associated with selective hereditary IgA deficiency. Dermatologica 1984;168:230-2.

3. Bennett ML, Jackson JM, Jorizzo JL, Fleischer AB Jr, White WL, Callen JP. Pyoderma gangrenosum. A comparison of typical and atypical forms with an emphasis on time to remission. Case review of $86 \mathrm{pa}-$ tients from 2 institutions. Medicine (Baltimore) 2000;79:37-46.

4. Wollina U. Pyoderma gangrenosum: a review. Orphanet J Rare Dis
2007;2:19.

5. Powell FC, Collins S. Pyoderma gangrenosum. Clin Dermatol 2000;18:283-93.

6. Su WP, Schroeter AL, Perry HO, Powell FC. Histopathologic and immunopathologic study of pyoderma gangrenosum. J Cutan Pathol 1986;13:323-30.

7. Weenig RH, Davis MD, Dahl PR, Su WP. Skin ulcers misdiagnosed as pyoderma gangrenosum. N Engl J Med 2002;347:1412-8.

8. Davis JC, Landeen JM, Levine RA. Pyoderma gangrenosum: skin grafting after preparation with hyperbaric oxygen. Plast Reconstr Surg 1987;79:200-7. 